\title{
Electron cloud simulations of a proton storage ring using cold proton bunches
}

\author{
Y. Sato, ${ }^{1,2,4}$ J. Holmes, ${ }^{1}$ S. Y. Lee, ${ }^{2}$ and R. Macek ${ }^{3}$ \\ ${ }^{1}$ Oak Ridge National Laboratory, Oak Ridge, Tennessee 37830, USA \\ ${ }^{2}$ Indiana University, Bloomington, Indiana 47405, USA \\ ${ }^{3}$ Los Alamos National Laboratory, Los Alamos, New Mexico 87545, USA \\ ${ }^{4}$ Nishina Center, RIKEN, Wako, Saitama, Japan \\ (Received 23 September 2007; published 11 February 2008)
}

\begin{abstract}
Using the ORBIT code we study the sensitivity of electron cloud properties with respect to different proton beam profiles, the secondary electron yield (SEY) parameter, and the proton loss rate. Our model uses a cold proton bunch to generate primary electrons and electromagnetic field for electron cloud dynamics. We study the dependence of the prompt and swept electron signals vs the bunch charge and the recovery of electron clouds after sweeping on the beam loss rate and the SEY. The simulation results are compared with the experimental data measured at the proton storage ring at the Los Alamos National Laboratory. Our simulations indicate that the fractional proton loss rate in the field-free straight section may be an exponential function of proton beam charge and may also be lower than the averaged fractional proton loss rate over the whole ring.
\end{abstract}

DOI: 10.1103/PhysRevSTAB.11.024201

PACS numbers: 29.27.Bd, 52.40.Mj, 52.35.Qz, 29.27.Fh

\section{INTRODUCTION}

In high intensity proton storage rings, the electron cloud effect (ECE) has been considered as one of the main sources of beam instability and emittance growth, which can lead to uncontrolled beam loss [1]. The importance of this subject is reflected in many international workshops in recent years. It is believed that the main source of the ECE is the trailing edge multipaction through secondary electron emission. Furman and Pivi have studied the secondary electron yield (SEY) of some materials that are commonly used for vacuum chambers in storage rings and have provided an algorithm for the calculation of electron cloud effects [2]. The algorithm has been used to study ECE of the proton storage ring (PSR) and at RHIC $[3,4]$.

In order to provide a more self-consistent treatment of particle beam dynamics, we develop an electron cloud simulation [5] with the popular accelerator code ORBIT $[6,7]$, that includes injection, accelerator lattice elements, closed orbit, space charge, impedance, etc. The ORBIT code is an open source code with parallel processing to simulate real machines for the design and analysis of high intensity accelerators.

This paper describes the simulation code and its application to understand the ECE experimental results in the PSR at Los Alamos National Laboratory. The simulation algorithm includes an electron cloud generation module $[8,9]$ using the Furman-Pivi algorithm but having a more flexible Monte Carlo scheme to reduce calculation time, and a beam dynamics module that deals with beam motion of the electron cloud and protons.

Generally, a proton bunch distribution has a mountainlike shape in the longitudinal coordinate. When the leading edge of a proton bunch passes through a region containing an electron cloud, the electrons in the vacuum chamber are trapped and made to oscillate inside the proton bunch. These electrons consist of carryover electrons that survived the beam gap and primary electrons produced by lost protons hitting the chamber surface. After the peak of the proton bunch passes the electron cloud region, these electrons are released from the proton bunch, hit the surface of the beam pipe, and cause secondary emission. The primary electrons produced in the trailing edge can typically gain enough energy to cause secondary emission upon hitting the wall. This mechanism is believed to be the main source of electron cloud buildup for a beam with long bunches in synchrotrons. In our simulations, we concentrate on the features of the electron cloud itself but not on the proton beam. Thus, we simulate the e-p problems with fixed proton bunch distributions, namely cold proton bunch. The electron cloud does not feedback onto proton motion. Since the electron cloud data to be analyzed were taken below the electron cloud instability threshold, the initial proton bunch profile is determined by the injection scheme, the rf voltage, the space charge force, and the inductive inserts $[7,10]$. The space charge force on protons is not important in our study.

The simulated electron cloud region has no applied magnetic field in order to be compared with experimental data, observed in an electron detector in a straight section at the PSR. First, we investigate the dependence of the electron cloud growth on the longitudinal triangular-shape proton distribution functions. The kicks of electric potential that drive primary electrons and generate secondary electrons are proportional to the slope of the proton bunch distribution. The energy distribution of electrons hitting the vacuum chamber surface depends also on the longitudinal proton distribution function. Second, we try to reproduce the electron cloud buildup data experimentally observed in the PSR [11]. The electron cloud data can be measured 
TABLE I. Physics and numerical parameters used in ORBIT simulation.

\begin{tabular}{lcc}
\hline \hline \multicolumn{1}{c}{ Parameter } & Symbol (unit) & PSR \\
\hline Ring parameters & & \\
Proton beam kinetic energy & $E(\mathrm{GeV})$ & 0.797 \\
Typical bunch charge & $Q_{\mathrm{p}}(\mu \mathrm{C})$ & $\sim 8$ \\
Ring circumference & $C(\mathrm{~m})$ & 90 \\
Revolution period & $T(\mathrm{~ns})$ & 358 \\
Bunch full length & $\tau_{\mathrm{b}}(\mathrm{ns})$ & 254 \\
Gaussian transverse beam size & $\sigma_{x}, \sigma_{y}(\mathrm{~mm})$ & 10,10 \\
Beam pipe radius & $a(\mathrm{~cm})$ & 5 \\
\hline Simulation physics parameters & & $\leq 4$ \\
Averaged fractional proton loss rate & $p_{\text {loss }}\left(10^{-6} / \mathrm{turn}\right)$ & $Y$ \\
Proton-electron yield & $\delta_{\max }$ & 100 \\
Maximum secondary yield & & 2.0 \\
\hline Numerical parameters & & 128 \\
Number of beam slices & & 1500 \\
Time steps in EM fields calculation/turn & & 20 \\
Tracker steps of electron motion/time step & & \\
\hline \hline
\end{tabular}

during the passage of the beam gap. The signal of swept electrons, defined as the electron current observed with an applied high voltage to detecting electrodes, shows saturation at high proton beam current. The signal of prompt electrons, defined as the electron current observed at the electron cloud detector after the passage of the beam without applying any voltage to the electrodes, shows no saturation. The electron cloud recovery time, after clearing the electrons by the high voltage sweep, provides another piece of information for our study.

To fit these data, free parameters in our simulations are the fractional proton loss rate $p_{\text {loss }}$ and the maximum SEY $\delta_{\max }$. The overall fractional proton loss rate has been experimentally measured in each turn but not at the electron cloud detector region. We use the ORBIT simulation code to fit experimental data, and thus estimate $p_{\text {loss }}$ in a drift space and $\delta_{\max }$. All physics parameters and experimental data used in this paper are taken from the PSR (see Table I).

We organize our paper as follows. In Sec. II, we describe the simulation model. In Sec. III we study the dependence of the electron cloud growth for the triangular bunch distribution. Here, we choose $\delta_{\max }=2.0$ and $p_{\text {loss }}=4 \times$ $10^{-6} /$ turn. In Sec. IV, we examine parameters needed to fit the prompt and swept electron cloud experimental data, and in Sec. V, we study parameters to fit the electron cloud recovery data. In these two sections, the parameters $\delta_{\max }$ and $p_{\text {loss }}$ will be treated as variables in order to fit the experimental data. The conclusion and discussion are discussed in Sec. VI.

\section{SIMULATION MODEL}

In our model, we assume a constant electron yield $Y$ per lost proton as is done in Ref. [4]. Because the prompt and swept electron data were taken at the stable proton beam condition, we use cold proton bunches to study the features of electron cloud generation, and we do not study the dynamics of the proton beam.

Since the electron cloud detector at the PSR is located in a straight section, the simulated electron cloud regions have no applied magnetic field. The longitudinal motion of the electrons is neglected for simplicity. In the longitudinal direction, each electron cloud region is distributed in a single $2 \mathrm{D}$ lattice, where the $2 \mathrm{D}$ mesh size is $64 \times 64$ for $100 \mathrm{~mm}$ diameter of vacuum chamber and the number of macroprotons in each longitudinal profile is 500000 . Two sources of electron cloud studied in this paper are (1) lost protons producing primary electrons, and (2) trailing edge multipaction from secondary electron emission. Experiments at the PSR showed that changing the vacuum pressure by an order of magnitude had no effect on beam stability. Thus, the effect of beam gas scattering on the electron cloud is small. The electron cloud studied in this paper is generated either by the primary proton loss or the secondary emission by trailing edge electron multipaction. The electrons trapped in the magnetic field region can become a very important primary electron source for the formation of an electron cloud in an adjacent straight section.

After the proton bunch passes the electron cloud region, the average line density of newborn primary zero energy electrons produced on the surface of the vacuum chamber is

$$
n_{\mathrm{e}, \mathrm{p}}=Y \times p_{\text {loss }} \times N_{\mathrm{p}},
$$

where $Y$ is the electron yield per lost proton, $p_{\text {loss }}$ is the fractional proton loss rate, and $N_{\mathrm{p}}$ is the line density of proton bunch. The primary electrons and the carryover 
electrons lead to trailing edge multipaction through a modeled secondary emission process on the surface of the vacuum chamber. We adopt the physics model of the stainless steel vacuum chamber surface used in Refs. [2,9]. To change $\delta_{\max }$ away from a value of 2, we modified the ORBIT secondary emission model [9] in such a way that the SEY $\delta\left(E_{0}\right)$ as a function of the incident electron energy $E_{0}$ can be set to a given $\delta_{\max }$ while the zero energy value, $\delta\left(E_{0}=0\right)=0.5$, is held fixed at 0.5 [4]. In our model, we change $\delta_{\max }$ while keeping the peak position in the energy range $E_{\max }=286 \sim 292 \mathrm{eV}$, which is the value of $E_{0}$ at which $\delta\left(E_{0}\right)=\delta_{\max }$.

\section{THE PROTON BUNCH SLOPE DEPENDENCE OF ELECTRON-CLOUD GROWTH AND ENERGY DISTRIBUTION OF ELECTRONS HITTING THE VACUUM CHAMBER SURFACE}

In this section, we study the behavior of the electron cloud due to the longitudinal shapes of cold proton bunches. We consider triangular density shapes for proton bunches in the longitudinal proton coordinate, where each bunch consists of a leading and a trailing edge. We arrange two types of longitudinal distributions: (1) triangles with a fixed leading slope but varying trailing slopes, and (2) triangles with varying leading slopes but a fixed trailing slope.

When the leading edge of the proton bunch passes through the electron cloud region, the proton bunches of the same leading slope accumulate the same number of primary electrons, while the trailing edge slope determines the energy gain of these electrons upon release from the bunch. On the other hand, proton bunches having different leading edge lengths trap different amounts of primary electrons before their peaks pass the electron cloud region. The same trailing edge slope gives rise to nearly the same energy distribution for the incident and secondary electrons at the surface of the beam pipe.

\section{A. Proton bunches of the same leading slope and the effect of the bunch trailing slope}

We consider proton pulses having the same line density in their $180 \mathrm{~ns}$-long heads but having different trailing edge slopes. Figure 1 shows that a longer proton bunch tail gives a higher electron cloud peak density, a later peak, and a higher carryover density of electron clouds. Since the electron cloud line density is nearly the same as the carryover density from previous turn at the front of the proton bunch and the carryover density depends on gap length between bunches, it is preferable to have a beam with a short trailing edge to reduce the ECE $[4,12]$.

The initial growth of the electron cloud signal in Fig. 1 is exponential. We define the electron cloud growth rate as the exponent of the electron cloud growth function at the time for the electron cloud density about halfway to its peak value. Figure 2 shows the growth rate of the electron

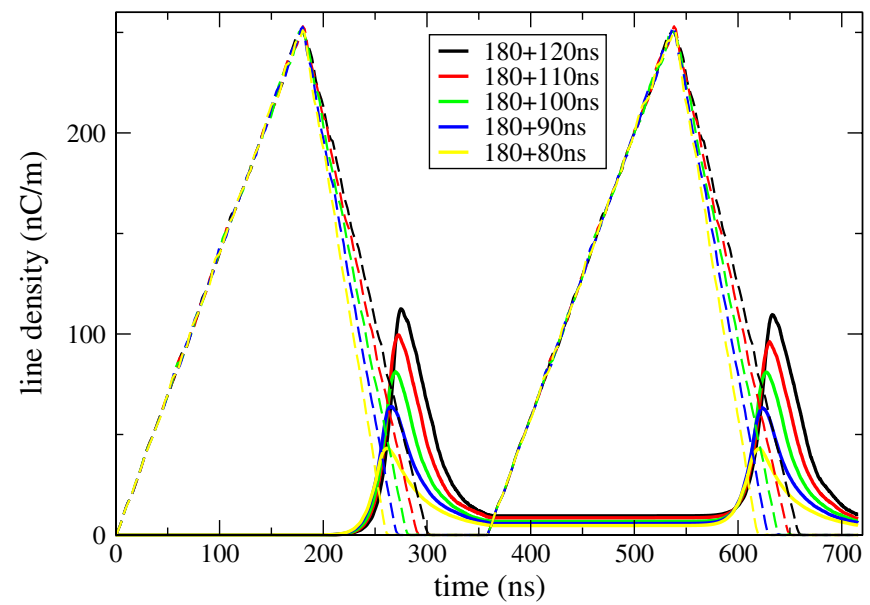

FIG. 1. (Color) The simulated electron cloud development of the same leading edge proton bunches. The solid lines are the line densities of electron clouds for the corresponding proton line densities shown as dashed lines. The leading edge length of the triangular proton bunches is $180 \mathrm{~ns}$. Each proton bunch holds $3.6 \times 10^{13}$ protons in the first $180 \mathrm{~ns}$.

cloud as a function of the proton bunch trailing slope. Note that the electron cloud growth rate becomes larger for a longer proton bunch tail in general. The first turn data corresponds to the electron cloud effect without the surviving electrons in the gap. The second turn electron cloud has a slightly lower growth rate probably because the surviving electrons can cause partial neutralization of the proton beam and reduce the multipacting process in the generation of the secondary electrons.

Now, we examine the energy distribution of the electron cloud. Figure 3 shows the energy distribution of electrons

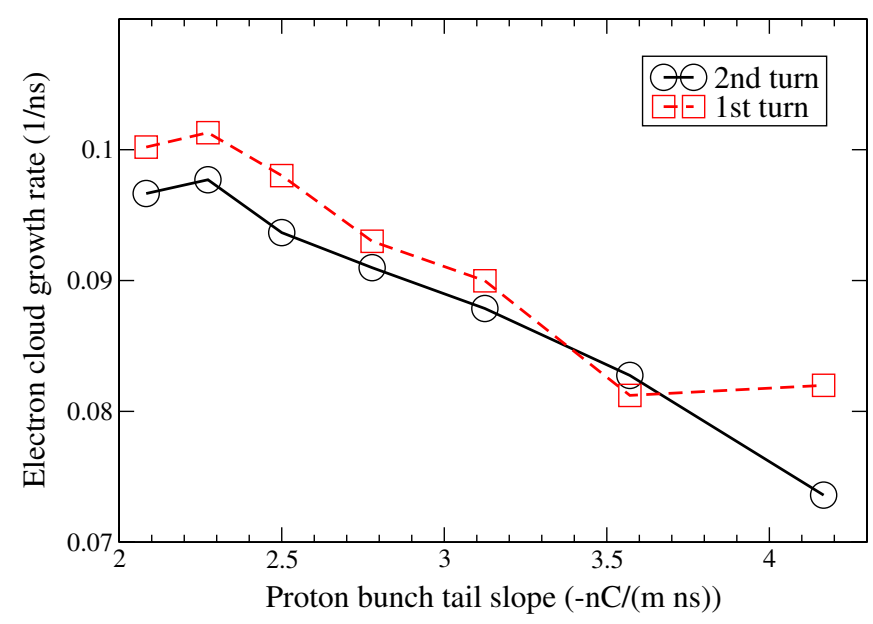

FIG. 2. (Color) The simulated growth rates of electron clouds for triangular bunch profiles with different trailing edge slopes in $[\mathrm{nC} /(\mathrm{m} \mathrm{ns})]$. The growth rate is calculated at the half height of the electron cloud line density. The red dashed line corresponds to the growth rate at the first turn, and the black solid line to the second turn. The growth rate of electron cloud becomes larger for a longer trailing edge, i.e., a smaller slope. 


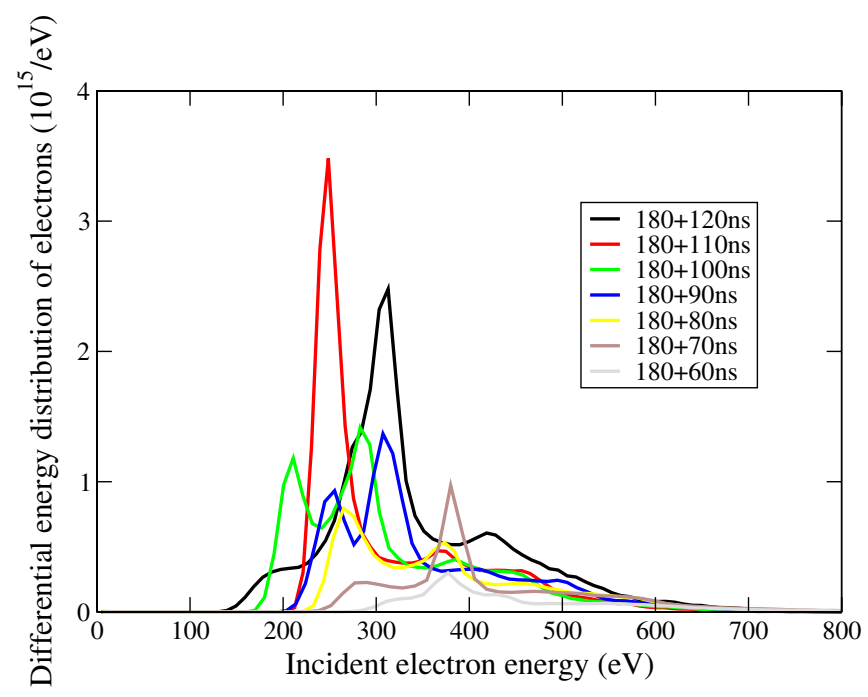

FIG. 3. (Color) The simulated energy distribution of electrons hitting the surface of the beam pipe at the half height of electron cloud line densities at the first turn for proton bunches with the same leading edge triangular proton profiles.

hitting the surface of the beam pipe for the first turn. Note that the steeper tail of the proton bunch gives a higher energy of electrons hitting the surface. However, the SEY decreases when the energy of electrons hitting the surface exceeds $300 \mathrm{eV}$. We need to remind ourselves that Figs. 2 and 3 are taken during the proton bunch tail passing the electron cloud region and just before the beam gap. Thus, the electron absorption and dissipation during the beam gap does not affect our discussion above. In summary, we find that a steeper beam trailing edge gives a smaller electron cloud, lower electron cloud growth, and higher energy of electrons hitting the surface.

\section{B. Proton bunches of the same trailing edge slope on the primary electron intensity}

We consider three proton pulses of the same 100 ns-long tails and the same maximum longitudinal line density but having different leading edge lengths of $160 \mathrm{~ns}, 180 \mathrm{~ns}$, and $200 \mathrm{~ns}$. The corresponding bunch charges are $(5.2,5.4$, 5.6) $\times 10^{13}$ protons, respectively. We focus on the effect of the primary electrons ahead of the bunch pulse peak. The fractional proton loss rate parameter $p_{\text {loss }}$ can be used to control the number of primary electrons. The effect of varying $p_{\text {loss }}$ will be discussed in Sec. V. In this section, we use the parameter $p_{\text {loss }}$ given in Table I.

Figure 4 shows that a longer leading edge will trap more carryover electrons due to a shorter gap. The high number of trapped electrons will produce a slightly larger electron cloud density. Since the carryover electrons trapped at the front of the proton bunch cannot escape the proton bunch until the very end of the trailing edge, they have less effect on the peak of the electron cloud density.

We also note that the growth rate of the electron cloud is less sensitive to the length of the proton bunch leading edge

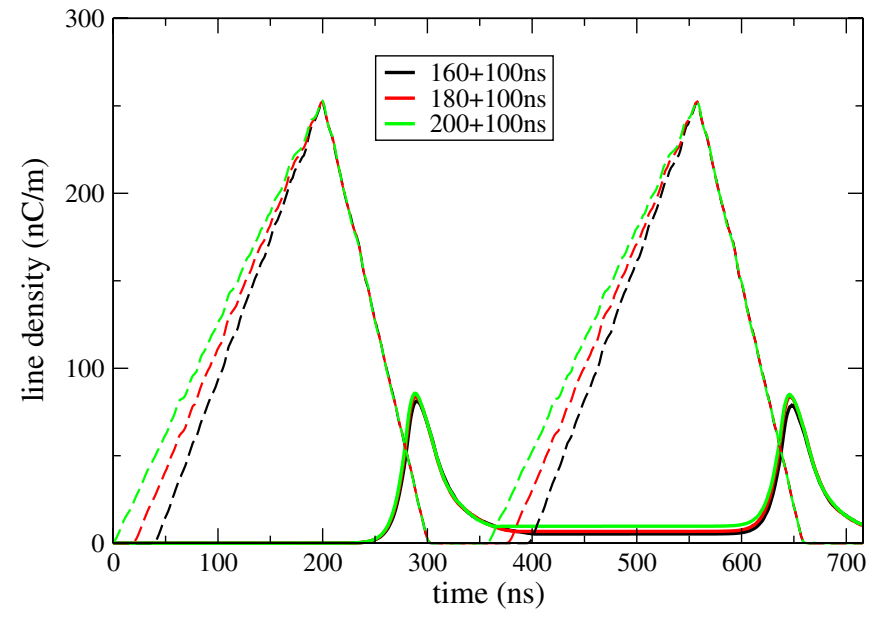

FIG. 4. (Color) The simulated electron cloud line densities due to proton bunches with the same trailing edge. The solid lines are the line densities of electron clouds for each corresponding proton bunch. The dashed lines are the line densities of proton bunch profiles with $100 \mathrm{~ns}$ triangular tail. Each proton bunch holds $2.0 \times 10^{13}$ protons in the $100 \mathrm{~ns}$ tail and (5.2, 5.4 , or 5.6) $\times 10^{13}$ protons as a whole. The same color relates each proton bunch profile to its corresponding electron cloud line density.

as shown in Fig. 5, where the scale is much smaller than that of Fig. 2. The carryover electrons have small influence on the electron cloud growth rate. This is consistent with the results of Fig. 2. The growth rate of the first turn provides information on the electron multipacting process.

Figure 6 shows the energy distribution of electrons hitting the surface of the beam pipe at the time that the proton beam exits the electron cloud region. We find that the energy distributions of secondary electrons are not very

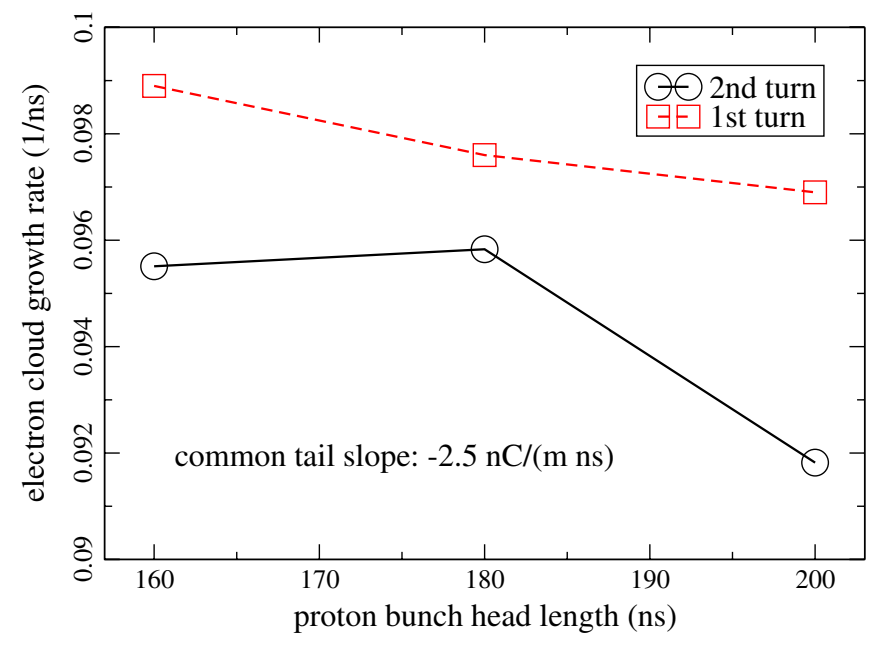

FIG. 5. (Color) The simulated growth rates of electron clouds for the same trailing edge triangular proton bunches. The growth rate is calculated at the half height of the electron cloud line density. The red dashed line corresponds to the growth rate at the first turn, and the black solid line for the second turn. 

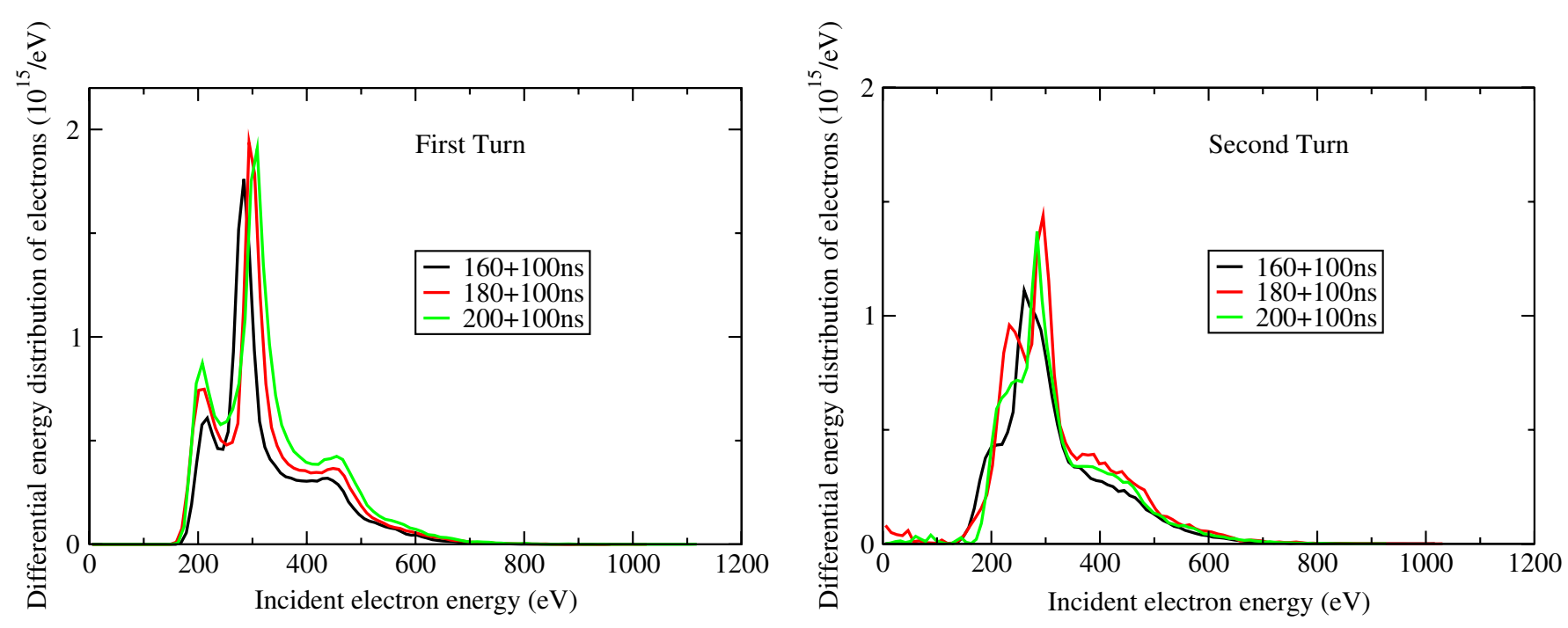

FIG. 6. (Color) The simulated energy distribution of electrons hitting the surface of the beam pipe at the half height of electron cloud line densities for proton bunches having the same trailing edge triangular profiles. The left plot is for the first turn, and the right plot is for the second turn.

sensitive to the bunch head length. In comparison with that of Fig. 3 of varying proton bunch tail slope, the energy of electrons hitting the surface, namely, the secondary electron emission process, is mostly determined by the proton bunch tail slope.

Each of the three proton bunches in this section has a corresponding proton bunch in Sec. III A holding the same charge. The (180 ns head, $120 \mathrm{~ns}$ tail), (180 ns head, $100 \mathrm{~ns}$ tail), and (180 ns head, $80 \mathrm{~ns}$ tail) proton bunches in Sec. III A correspond to the (160 ns head, $100 \mathrm{~ns}$ tail), (180 ns head, $100 \mathrm{~ns}$ tail), and (200 ns head, $100 \mathrm{~ns}$ tail) proton bunches in this section, respectively. Since each pair has the same bunch charge, it generates the same number of total primary electrons. Comparing the results of these three pairs, we find that the peak height and the growth rate of the electron cloud depend more sensitively on the electric potential and the primary electrons during the passage of the proton bunch trailing edge than on the carryover electrons and primary electrons during the passage of the proton bunch leading edge. Thus, having a long leading edge in the proton bunch profile is a possible way to accumulate more protons without changing the electron cloud intensity. However, from the point of view of e-p centroid oscillation [9], more carryover electrons can make the proton bunch more unstable, cause more proton losses, and in the end, generate a larger electron cloud.

\section{SIMULATION AND COMPARISON OF THE PROMPT AND SWEPT ELECTRONS}

In this section we compare our simulation results with the experimental data measured at the PSR [11] vs the proton bunch charge. The prompt and the swept electron signals are taken at the same electron sweeper located in a straight section in PSR. The electron sweeper can sweep most of the electrons inside the vacuum chamber when a high voltage (HV) pulse is applied after the proton bunch passed through the detector area. Without the application of HV pulse, it is an electron detector that counts surface electron current from the chamber to its collector. The prompt electron signal is defined as the peak height of the electron signal without HV pulse in the steady state. The swept electron signal is the height of the electron cloud signal after the application of a HV pulse sweep.

In our simulation, the incident electron flux onto the vacuum chamber surface is the prompt electron signal. Also, the electron cloud line density at the head of the succeeding proton bunch can be treated as the swept electron signal, because the measured swept electron signal corresponds to the whole charge of the electron cloud inside the chamber at the detector area. The simulation parameters are listed in Table I. Here the mesh size for the proton beam transverse cross section is 128 , the number of time steps for the electromagnetic field calculation in each slice is 1500 , and the motion of electrons in each time step is calculated in 20 integration steps. However, we vary $p_{\text {loss }}$ and $\delta_{\max }$ to reproduce the experimental data. The dependence of SEY as a function of the electron cloud energy has been described in Sec. II.

In order to understand prompt and swept electron signals, we divide this section into three subsections. In Sec. IVA, both $p_{\text {loss }}$ and $\delta_{\max }$ are held constant independent of bunch charge. In Sec. IV B, $p_{\text {loss }}$ is taken as a function of bunch charge. In Sec. IV C, we study also the effect of longitudinal beam profile.

\section{A. Simulations with constant fractional proton loss rate}

In this section, we assume that the fractional proton loss rate is independent of the bunch charge. Namely, we 

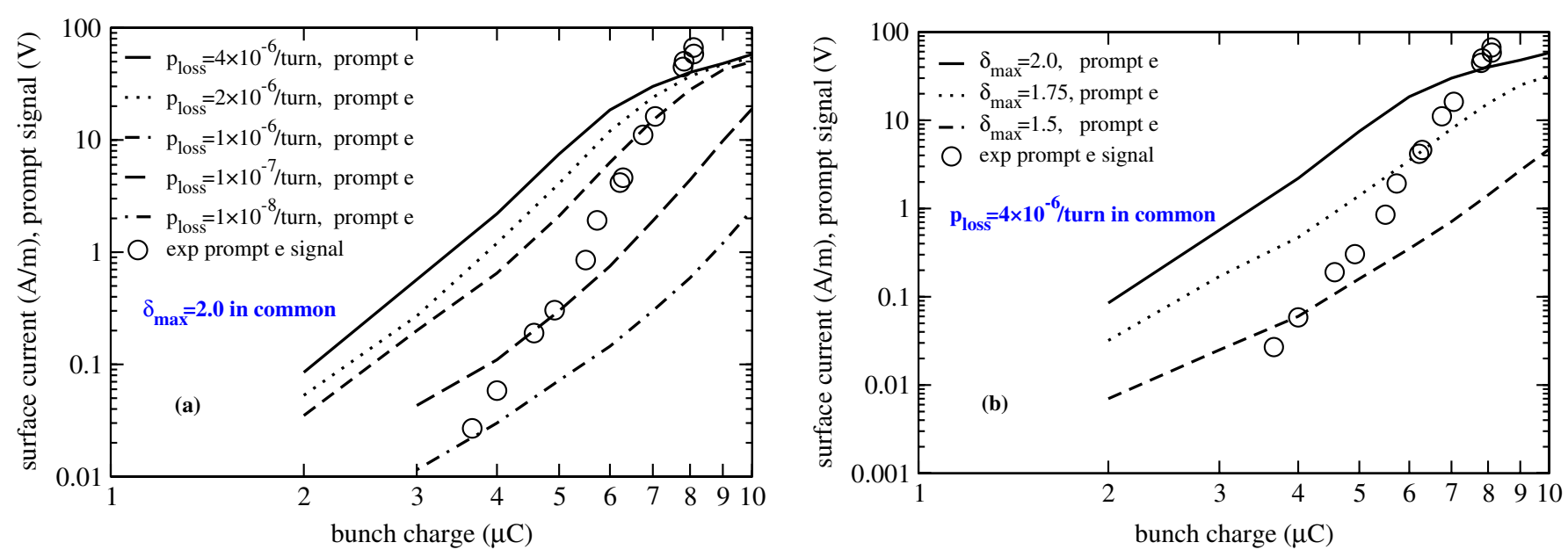

FIG. 7. (Color) The signal of prompt electrons, simulated incident current at the surface in $\mathrm{A} / \mathrm{m}$, is compared with the experimental signal data in volts (V). The prompt signal vs the fractional proton loss rate at a constant $\delta_{\max }=2.0$ is shown in (a). The prompt signal with varying SEY with a constant $p_{\text {loss }}=4.0 \times 10^{-6} /$ turn is shown in (b). The lines are the simulated peak heights of surface current in $(\mathrm{A} / \mathrm{m})$ of the electron cloud. The circles mark experimental data of peak heights of electron signal in units of volts.

assume a constant rate of seed-electron production, hence the amount of primary electrons is proportional to the bunch charge. Figure 7 shows the simulated incident electron current at the chamber surface, i.e., the prompt electrons, as a function of the proton bunch charge. Experimental data are shown as circle symbols. The left plot shows prompt electron intensity for $p_{\text {loss }}$ varying from $1 \times$ $10^{-8} /$ turn to $4 \times 10^{-6} /$ turn with a fixed $\delta_{\max }=2$. The right plot shows the prompt electron intensity for $\delta_{\max }$ varying from 1.5 to 2.0 while keeping a constant $p_{\text {loss }}=$ $4 \times 10^{-6} /$ turn. We note that the simulated functional dependence on the bunch charge differs substantially from that of the experimental data.

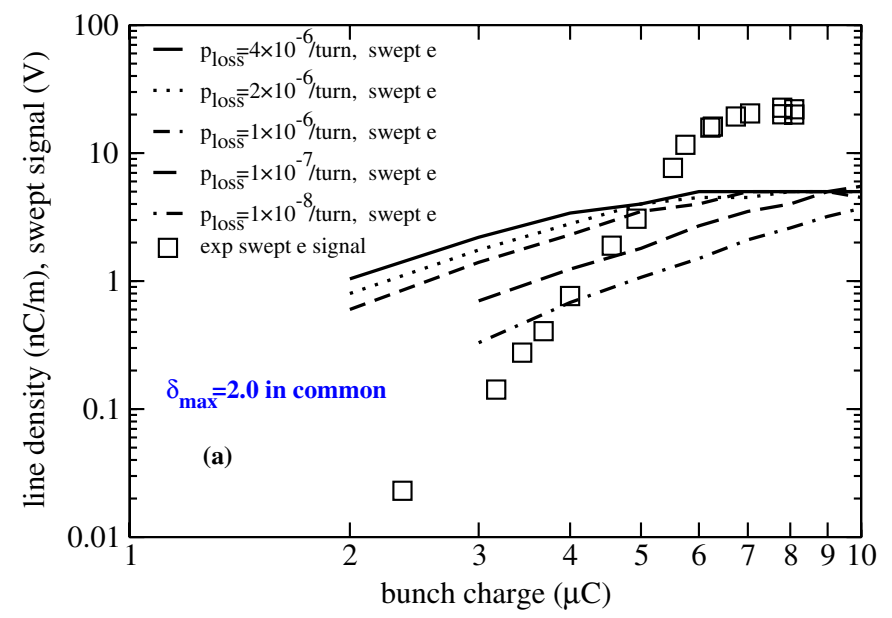

Figure 8 shows the simulated electron cloud line density at the beginning of the proton beam pulse or equivalently at the end of the beam gap, i.e., the swept electrons, as a function of the proton bunch charge. Experimental data are shown as rectangle symbols. Plot (a) shows prompt electron intensity for $p_{\text {loss }}$ varying from $1 \times 10^{-8} /$ turn to $4 \times$ $10^{-6} /$ turn with a fixed $\delta_{\max }=2$. Plot (b) shows the prompt electron intensity for $\delta_{\max }$ varying from 1.5 to 2.0 while keeping a constant $p_{\text {loss }}=4 \times 10^{-6} /$ turn.

We note that the slopes of the simulated prompt and swept signals vs the bunch charge are much smaller than those of the experimental data. If we use either $\delta_{\max }=2.0$ or $p_{\text {loss }}=4.0 \times 10^{-6} /$ turn for all bunch charges, we can-

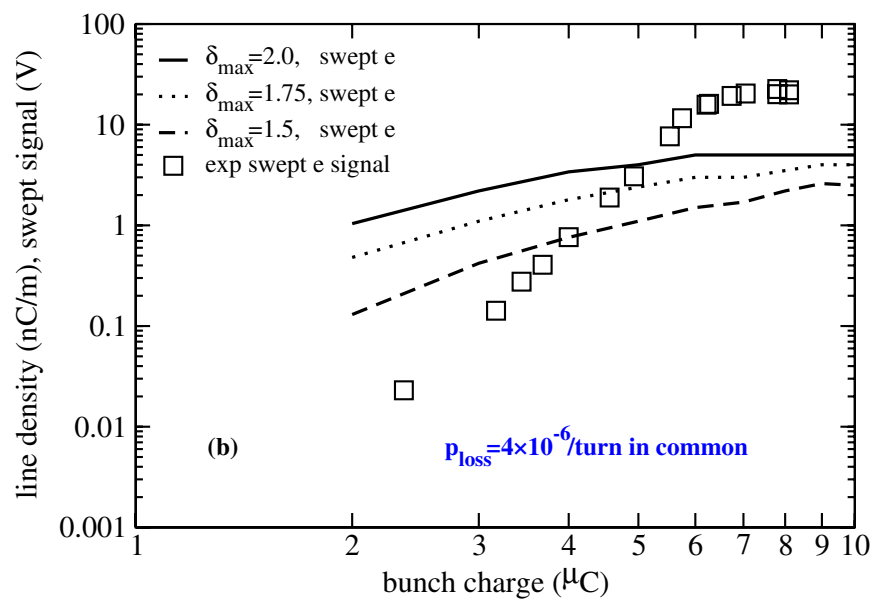

FIG. 8. (Color) The signal of the swept electrons, simulated electron line density in $(\mathrm{nC} / \mathrm{m})$, is compared with the experimental swept signal in volts $(\mathrm{V})$. The swept signal for various fractional proton loss rates at a constant $\delta_{\max }=2.0$ is shown in (a). The swept signal with varying maximum SEY at a constant $p_{\text {loss }}=4.0 \times 10^{-6} /$ turn is shown in (b). The lines are the simulated electron line densities $(\mathrm{nC} / \mathrm{m})$ when heads of proton pulses enter the electron cloud region. The square marks are experimental data of peak heights of the swept electron signal in volts. 
not fit the measured prompt and swept electron signals. For constant $\delta_{\max }$ and $p_{\text {loss }}$ vs the bunch charge, the simulated slopes are less steep than the experimental slopes for both prompt and swept electrons. Lowering either $\delta_{\max }$ or $p_{\text {loss }}$, simulated prompt and swept electrons are reduced. The simulated prompt and swept slopes become slightly steeper but still much flatter than the slopes of the experimental data.

The experimental swept electron slope has a special feature that there is a saturation for the high bunch charge at about $6 \mu \mathrm{C}$. This feature is reproduced only for higher secondary emission yield $\delta_{\max }$ and higher fractional proton loss rate $p_{\text {loss }} \approx 4.0 \times 10^{-6} /$ turn, the averaged value over the whole ring. A simulation of prompt and swept electrons using SNS parameters is performed by Wang et al. [3]. Their results also reproduce the qualitative feature of saturation of swept electron slope for the high bunch charge at about $5.0 \mu \mathrm{C} /$ pulse and $p_{\text {loss }}=1.1 \times 10^{-6} /$ turn, consistent with our results.

\section{B. Simulations with varying fractional proton loss rate vs the bunch charge}

In Sec IVA, we assumed that $\delta_{\max }$ and $p_{\text {loss }}$ were independent of the bunch charge. However, this assumption cannot reproduce experimental results. It is very likely that $p_{\text {loss }}$ depends on bunch charge, but this dependence is less likely for $\delta_{\max }$. This assumption sounds reasonable because higher bunch charge may have higher beam loss rate due to beam dynamics issues. We however assume a constant $\delta_{\max }$, which depends on beam pipe conditions such as scrubbing, vacuum condition, etc. Thus, we assume that $p_{\text {loss }}$ is a function of bunch charge, i.e., the seedelectron production rate depends on bunch charge. We perform simultaneous fits of both the experimental prompt and swept slopes using the single fitting parameter $p_{\text {loss }}$.

The fitting procedure is as follows: (i) We assume $\delta_{\max }=1.7$. (ii) The simulated prompt and swept values of $p_{\text {loss }}=4.0 \times 10^{-6} /$ turn for bunch charge $7 \mu \mathrm{C} /$ pulse are set as the reference point. (iii) For a given bunch charge $Q_{\mathrm{p}}$, we find the parameter $p_{\text {loss }}$ such that the prompt and swept signals fit both experimental prompt and swept slopes. (iv) The fractional proton loss rate function $p_{\text {loss }}\left(Q_{\mathrm{p}}\right)$ is determined by repeating the step (iii).

Figure 9 shows the slope fitting of both simulated prompt and swept data. We note that the resulting proton loss function $p_{\text {loss }}\left(Q_{\mathrm{p}}\right)$ is nearly exponential. There are some remaining questions about the assumption of $p_{\text {loss }}$ as a function of bunch charge. The simulated beam of $4 \mu \mathrm{C} /$ pulse in Fig. 9 has maximum proton beam line density $160 \mathrm{nC} / \mathrm{m}$ and electron cloud peak line density $0.092 \mathrm{nC} / \mathrm{m}$. This amount of electron cloud may be too small to cause proton beam instability, though the instability is measured even for a beam of less $4 \mu \mathrm{C} /$ pulse at PSR. In the future, we need to study the origin and location of fractional proton loss rate function, and perform simulations to check centroid oscillations of proton beam with small fractional proton loss rate parameter. We need to remind ourselves that there are different sources of primary electrons. Therefore, the functional dependence of $p_{\text {loss }}\left(Q_{\mathrm{p}}\right)$ may reflect the unknown electron sources at high bunch charge.

\section{Beam profile dependence}

Figure 10 shows the dependence of prompt and swept electrons on realistic proton bunch profiles, where the nonotched beam is produced by injecting linac beamlets into
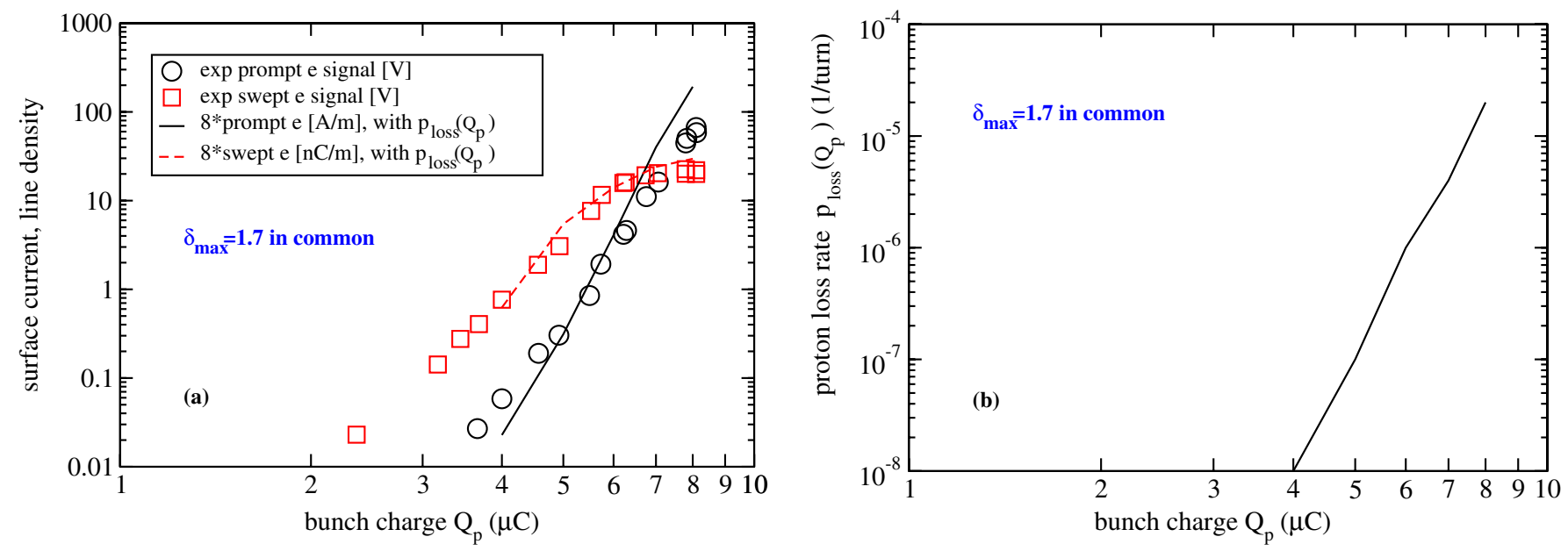

FIG. 9. (Color) Using a constant $\delta_{\max }=1.7$, we fit the prompt and swept simulated data with varying fractional proton loss rate $p_{\text {loss }}\left(Q_{\mathrm{p}}\right)$ as a function of bunch charge $Q_{\mathrm{p}}$ in (a). Note that the simulated prompt surface current line density and sweep charge line density are normalized by multiplying a factor of 8 to match the scale of data in volts. The resulting fractional proton loss rate is shown in (b). 

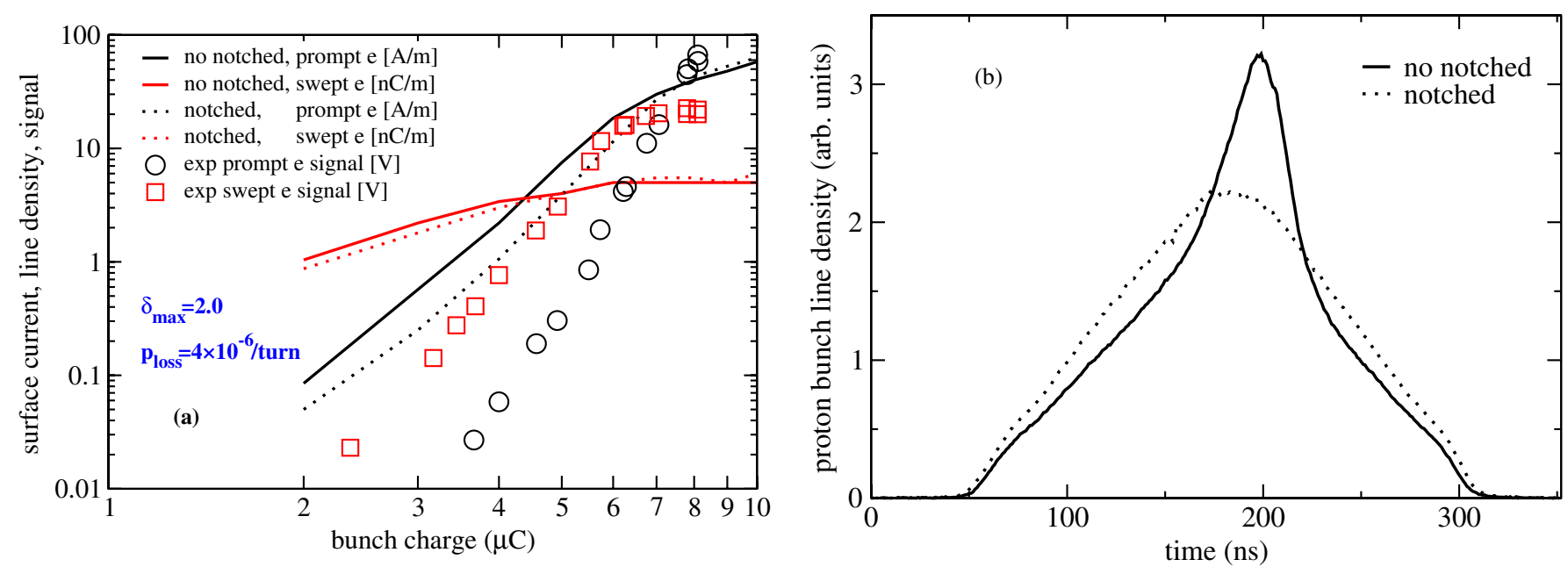

FIG. 10. (Color) The simulated prompt and swept electron signals for the no-notched and notched proton pulses are shown in (a) with a constant $\delta_{\max }=2.0$ and $p_{\text {loss }}=4.0 \times 10^{-6} /$ turn. The notched proton profile corresponds to an injection with a chopped beam at the center of the bucket in order to minimize the peak proton beam current, while the no-notched proton profile refers to the injection without the chopper. The black lines and plots correspond to prompt electrons. The red lines and plots are swept electrons. The lines are ORBIT simulation results, where the solid lines correspond to no-notched proton pulse, and the dotted lines notched proton pulse. The measured experimental plots are for no-notched proton pulses. The longitudinal profiles of no-notched (solid line) and notched (dotted line) proton pulses are shown in (b).

the PSR without phase space painting, and the notched beam corresponds to injected beamlets with a small beam gap in the middle of the bunch center. The notched beam has a smaller peak current at the center of the bunch. The notched profile has less electron cloud development than the no-notched profile for the same amount of bunch charge below $6 \mu \mathrm{C} /$ pulse. On the other hand, the notched profile produces more electron cloud at high charge, i.e. $>8 \mu \mathrm{C} /$ pulse. This is a consequence of the notched beam profile having a longer tail.

In Secs. IVA and IV B, the no-notched longitudinal beam profile is used in all simulations, but the actual profile can be different among beam intensities. However, Fig. 10(a) indicates that the profile difference does not change prompt and swept electron slopes dramatically. Thus, the actual profile difference may not solve the problem discussed in Sec. IVA.

\section{ELECTRON CLOUD RECOVERY SIMULATIONS}

In PSR, it has been experimentally observed that the peak signal of the electron cloud is reduced substantially following the clearing of electrons in the gap by the action of the electron sweeper device. The electron cloud takes several turns to recover [11]. Figure 11 shows that the electron cloud in the case of the $7.5 \mu \mathrm{C}$ bunch current needs 5 turns to recover. We can expect that most of the captured electrons that survived in the bunch gap are swept away from the beam pipe. Thus, we assume in the simulation that there are no electron clouds before the first turn, and we follow the surviving electrons from the previous gap turn by turn. We apply electron cloud simulations as a function of $Q_{\mathrm{p}}, p_{\text {loss }}$, and $\delta_{\max }$ in the area of the electron sweeper. In general, lower $Q_{\text {loss }}, p_{\text {loss }}$ and $\delta_{\max }$ are necessary in order to increase the number of recovery turns.

\section{A. Fractional proton loss rate estimation}

Figure 11 shows the recovery process of the electron cloud measured at the PSR. We note that it takes 4-5 turns for the electron cloud to reach its steady state intensity. Figure 12 shows the electron cloud recovery with a constant $\delta_{\max }=2.0$ and varying $p_{\text {loss }}$. For a first turn recovery, we estimate that $p_{\text {loss }}$ for a $7.5 \mu \mathrm{C}$ beam in drift space is around $p_{\text {loss }}=1.0 \times 10^{-8} /$ turn at $\delta_{\max }=2.0$, or $p_{\text {loss }}=$ $1.0 \times 10^{-7} /$ turn for $\delta_{\max }=1.7$. There is also a possibility that $p_{\text {loss }}$ is a function of beam pulse charge as suggested in Sec. IV B. We need to reproduce the experimental recovery processes and get the estimated $p_{\text {loss }}$ for different bunch charges.

\section{B. Inconsistency between recovery estimation and prompt and swept slope fit}

From the prompt and swept electrons vs bunch charge study, we find that we need $p_{\text {loss }}=4.0 \times 10^{-6} /$ turn with $\delta_{\max }=1.7$ for $7.0 \mu \mathrm{C}$ bunch charge. Varying other parameters, we need $p_{\text {loss }}>1.0 \times 10^{-6} /$ turn to fit the prompt and swept data. The measured prompt and swept electron data indicate that there are a lot of seed primary electrons in the electron detector area.

On the other hand, from the recovery time study, we find that $p_{\text {loss }} \approx 1.0 \times 10^{-7} /$ turn $\sim 1.0 \times 10^{-8} /$ turn for the 


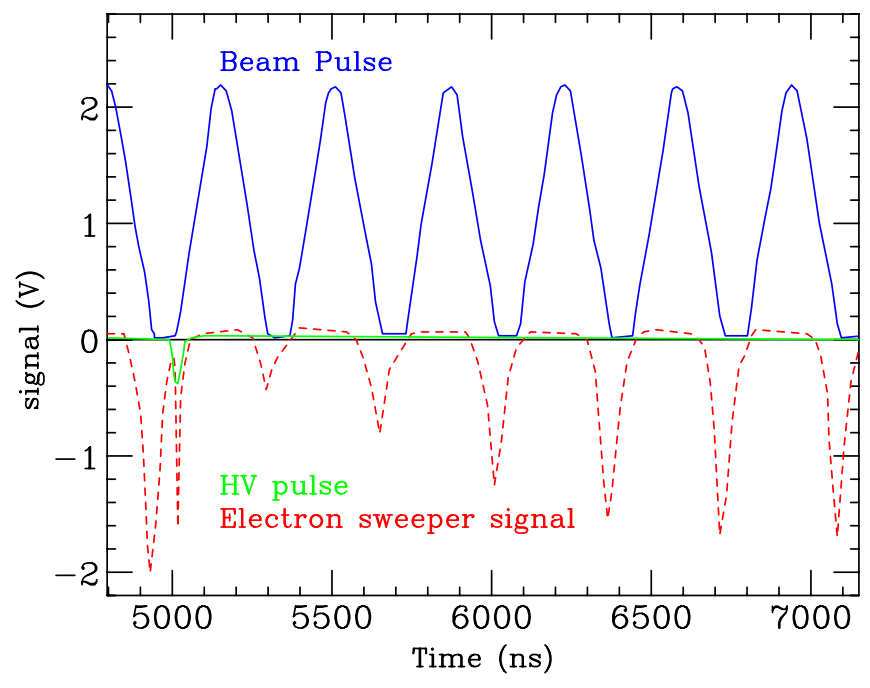

FIG. 11. (Color) The measured recovery process of electron cloud development in the PSR experiment. The proton bunch charge is $7.5 \mu \mathrm{C}$. The high voltage pulse applied on the electron sweeper is a green line. The red line is the signal of the electron sweeper. Without applying HV on the electron sweeper, we expect that its signal is proportional to the current of electrons absorbed in the beam pipe. After the electrons are swept out, the peak height of electron cloud signal needs 4 or 5 turns to recover. The swept electron signal is a spike after the HV is applied. From the structure of the electron sweeper device at PSR [11], we can expect that most of the captured electrons surviving bunch gap are swept away from the beam pipe.

$7.5 \mu \mathrm{C}$ beam. The recovery time experiments indicate that, once the seed electrons are swept away, it takes a few turns to recover these electrons. The inconsistency of estimated $p_{\text {loss }}$ between recovery study and prompt and swept slope study indicates that the seed electrons are not only generated by proton loss at the detector area. As stated in Sec. II, electrons do not move in the longitudinal coordinate in our simulations, and thus electrons outside the

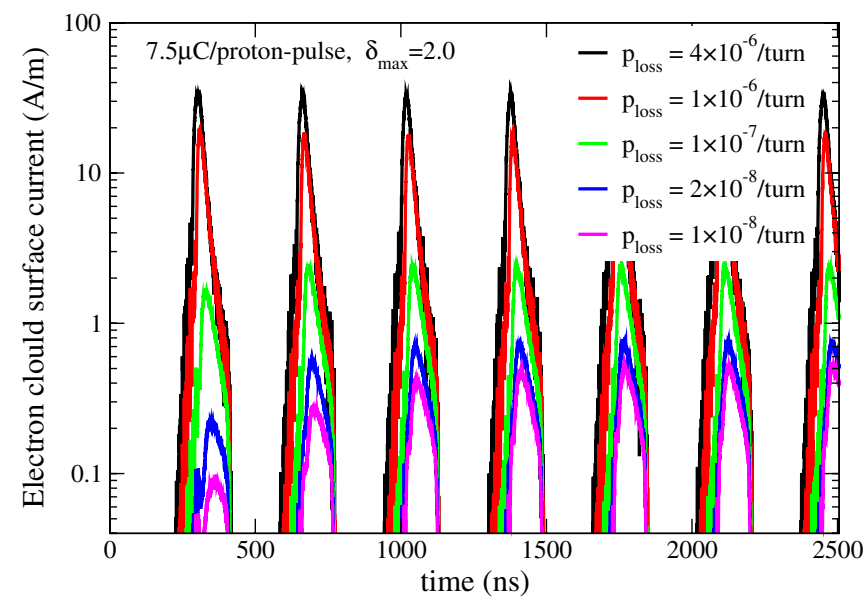

FIG. 12. (Color) The simulated surface current from electron cloud in a $7.5 \mu \mathrm{C}$ proton pulse for various fractional proton loss rates at a constant $\delta_{\max }=2.0$. simulated region are not considered in our model. Recent experimental results at PSR $[13,14]$ indicate that electrons can be ejected from quadrupole magnets into the drift space. Furthermore, the recovery time is found to be much longer when electron reflection mirrors are used to prevent electrons from the upstream and downstream quadrupoles for migrating to the field-free region containing the detector. If this is the case, the recovery essentially depends on the traveling time of electrons in magnetic field regions, and the slopes of the prompt and swept electron clouds depend essentially on the primary electrons in these regions. Including this effect may be a possible way to achieve the self-consistency between the recovery estimation and the assumption of fractional proton loss rate function.

We have also found that the energy distributions of electrons hitting the surface of vacuum chamber in 5 recovery turns are nearly identical even though the peak height of the electron cloud density increases turn by turn. Therefore, the energy distribution is mainly determined not by the amount of carryover electrons but by the longitudinal proton bunch profile discussed earlier. This is consistent with the result of Sec. III B, where we find that the energy spectrum depends essentially on the trailing edge slope of the proton bunch. The amount of carryover electrons affects the electron cloud development mostly during its recovery process after clearing gap.

\section{CONCLUSIONS}

We have examined electron cloud properties using the ORBIT code simulation of a cold proton beam bunch. We use the PSR parameters for our physics study and the simulated electron cloud region is located in a straight section, where there is no magnetic field.

In the simulations of triangular profile proton bunches, a longer proton bunch trailing edge gives a larger electron cloud, a larger growth rate, and a higher peak electron cloud current at the end of bunch tail. However, the primary and remnant electrons captured by the front leading edge of proton bunch have little effect on electron cloud development. We also find a beam profile of longer head is a possible way to accumulate more protons without making the electron cloud more intense.

In the prompt and swept electron study, the simulated prompt electron current shows a steeper slope than the simulated swept electron line density as functions of $Q_{\mathrm{p}}$. The simulated swept electron line density shows saturation for $Q_{\mathrm{p}}>6 \mu \mathrm{C}$. This is consistent with the experimental data. However, the simulated prompt and swept electron slopes are much smaller than the experimental data. If we treat fractional proton loss rate $p_{\text {loss }}$ as a function of $Q_{\mathrm{p}}$, we can fit prompt and swept electron slopes to experimental data. This fitted fractional proton loss rate is exponential vs the bunch charge. The space charge effect of a bunch is a possible reason. 
On the other hand, when we studied the electron cloud recovery time after the electron clearing beam gap, we found that $p_{\text {loss }}$ in a field-free straight section must be smaller than $1 \times 10^{-7} /$ turn. The apparent inconsistency of the prompt and swept data and the electron cloud recovery data imply that there are other possible electron sources in the straight section. We have also used the actual proton bunch profile of PSR for each bunch charge to study the prompt and swept slopes and the electron cloud recovery. Our results indicate that this inconsistency cannot be resolved by the actual beam profile.

Currently, there are efforts to measure the electron cloud in quadrupoles at PSR. The results indicate that there are copious electrons ejected from the quadrupoles into the adjacent straight section. Our current simulation model needs to be augmented to account for such electrons. A self-consistency model to explain the recovery time and the prompt and swept dependence on the bunch charge is needed. In our current simulation model, however, we cannot simultaneously perform the fit of the prompt and swept electron slopes and the fit of electron cloud recovery after sweeping. A simulation model of electrons traveling from the quadrupole magnet area to drift space may be a possible way to solve this inconsistency. To do so, we need to simulate electron cloud development including longitudinal dynamics, or to obtain experimental data of electron cloud drift speed from quadrupole magnets. We will continue to study this problem in the future.

\section{ACKNOWLEDGMENTS}

The authors would like to thank Andrei Shishlo and Slava Danilov for useful and helpful discussions. This research used resources of the NERSC, supported by the U.S. DOE under Contract No. DE-AC03-76SF00098. This work is supported by SNS through UT-Battelle, LLC, under Contract No. DE-AC05-00OR22725 for the U.S. DOE, by Indiana University at Bloomington under
Contract No. PHY-0552389 for NSF and Contract No. DEFG02-92ER40747 for DOE, and by Los Alamos National Laboratory under Contract No. W-7405-ENG-36.

[1] D. Neuffer, E. Colton, D. Fitzgerald, T. Hardek, R. Hutson, R. Macek, M. Plum, H. Thiessen, and T.S. Wang, Nucl. Instrum. Methods Phys. Res., Sect. A 321, 1 (1992).

[2] M. A. Furman and M.T.F. Pivi, Phys. Rev. ST Accel. Beams 5, 124404 (2002).

[3] L. Wang, M. Blaskiewicz, H. Hseuh, P. He, Y. Y. Lee, D. Raparia, J. Wei, S. Y. Zhang, and R. Macek, in Proceedings of the ECLOUD04, Napa, CA, 2004.

[4] M. T. F. Pivi and M. A. Furman, Phys. Rev. ST Accel. Beams 6, 034201 (2003).

[5] Y. Sato, Ph.D. thesis, Indiana University, Bloomington, 2006.

[6] J.A. Holmes, V. Danilov, J. Galambos, A. Shishlo, S. Cousineau, W. Chou, L. Michelotti, F. Ostiguy, and J. Wei, Proceedings of the EPAC02, Paris, France, 2002, p. 1022.

[7] S. M. Cousineau, Ph.D. thesis, Indiana University, Bloomington, 2002.

[8] A. Shishlo, Y. Sato, J. Holmes, S. Danilov, and S. Henderson, in Proceedings of ECLOUD04 workshop, Napa, CA, 2004.

[9] Y. Sato, A. Shishlo, J. Holmes, S. Danilov, and S. Henderson, in Proceedings of ECLOUD04 workshop, Napa, CA, 2004.

[10] C. Beltran, Ph.D. thesis, Indiana University, Bloomington, 2003.

[11] R. Macek, http://physics.indiana.edu/ shylee/ap/mwapc/ epfeedback.html.

[12] M. T. F. Pivi and M.A. Furman, Proceedings of PACO3 (IEEE, New York, 2003), p. 3222.

[13] R. Macek, http://physics.indiana.edu/ shylee/ap/mwapc/ epws.html.

[14] R. J. Macek et al., Proceedings of the PAC 2007 (2007), p. 828 . 